\title{
FANCONI'S SYNDROME
}

\section{Treatment by Massive Dosage of Calciferol and Two-stage Osteotomy}

\author{
Martin Singer, LoNdon, ENgland
}

From the Institute of Orthopaedics, Royal National Orthopaedic Hospital

Rickets caused by deficiency of vitamin D has almost disappeared in Great Britain, and for this reason attention is being focused more and more on the group of conditions which in the past has been loosely termed " resistant" and " renal " rickets.

Dent (1952) clarified the confusion that has surrounded the subject. He classified the etiology of the various rachitic and osteomalacic conditions into the following groups: 1) vitamin $\mathrm{D}$ and calcium lack; 2) the steatorrhoeas (coeliac, idiopathic, sprue); 3) bile salt deficiency: ? hepatic rickets; 4) renal glomerular defect (polycystic kidney and other gross renal defects) ; 5) renal tubular defect; ureterocolostomy.

In renal glomerular rickets, which is in fact the classical " renal rickets," both glomeruli and tubules are damaged or destroyed, leading to retention of urea, phosphates and other substances normally excreted in the urine.

In renal tubular rickets there is an inherited or acquired defect of tubular reabsorption. Various functions may be lost, depending on the site and extent of the defect. For clinical purposes this group can be sub-divided into six types, according to the nature of the tubular reabsorption defect: 1) phosphates only (low blood phosphorus); 2) phosphate+glucose (glycosuria); 3) phosphate + glucose + amino-acids (amino-aciduria, including cystinuria); 4) phosphate+glucose+amino-acids, with faulty urine acidification (low $\mathrm{CO}_{2}$ combining power); 5) phosphate, with faulty urine acidification and high urinary calcium content; 6) phosphate, with faulty urine acidification, defective ammonia formation, and high urinary calcium content.

Many names have been given to sporadic cases of these conditions in the past. For example, types 1 and 2 have been described as resistant rickets, essential hypophosphataemic rickets, and Milkman's syndrome; types 3 and 4 have been termed Fanconi's syndrome, cystine rickets, renal rickets, and hypophosphataemic glycosuric rickets; and types 5 and 6 are sometimes called nephrocalcinosis with renal rickets and dwarfism, or Butler-Albright's syndrome. Types 1 to 4 tend to run in families. On the other hand types 5 and 6 are never hereditary, the cause being probably not genetic but environmental.

These six varieties of tubular defect may occur in either sex and at any age and may show spontaneous remissions and exacerbations. Radiographically they may be indistinguishable from each other and thus the differential diagnosis rests with the biochemist rather than the clinician. It is important to recognise these conditions because the results of treatment are often gratifying.

The case reported here accords with Fanconi's syndrome (type 3 or 4 of the classification), the signs of which are considered by Dent (1952) to be due to an inherited defect of reabsorption affecting primarily the proximal tubules of the kidney and involving amino-acids, sugar and phosphates. Like Fanconi (1936) he believes the disease to be inherited as a Mendelian recessive character. Severe cases show dwarfism, gross rickets, hypophosphataemia, aminoaciduria (including cystinuria), glycosuria, deposition of cystine in body tissues, and acidosis. The urinary output of glucose and amino-acids often varies from day to day.

\section{GASE REPORT}

A boy - an only child - was born at full term and the delivery was normal. He was breast-fed for nine months and had cod liver oil from the age of one month. There was no 


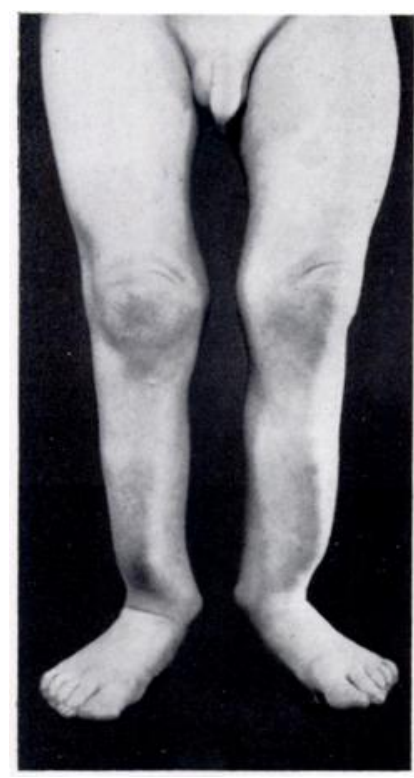

FIG. 1

Before treatment. Note marked bowing of tibiae.

feeding difficulty, undue thirst or vomiting, but his weight was below normal. He was first seen at the Royal National Orthopaedic Hospital at the age of sixteen months with florid rickets (Figs. 1, 2, 3, 5). A striking feature of his appearance was that he was completely bald except for a small tuft of hair on the vertex. The kidneys were not palpable. The radiographs were typical of severe rickets (Figs. 2, 3, 5). The renal shadows were normal in size and position.

Investigations (at the age of eighteen months) showed the following results. L'rine-There was an abnormal concentration of amino-acids (square chromatograms) (glycine, alanine, glutamine, lysine, and traces of cystine, threonine, valine and tyrosine). There was no glycosuria or albuminuria. Bloodserum calcium $9 \cdot 2$ milligrams per cent (within normal limits); alkaline phosphatase 73 units (normal :5-1:5); blood phosphorus $\mathbf{2} \cdot \mathbf{7}$ milligrams per cent (normal 5 for children); $\left({ }^{\circ} \mathrm{O}_{2}\right.$ combining power 39 vols. (normal 5i)-75); blood urea 29 milligrams per cent. Faeces- Normal fat analysis. Slit-lamp examination-. No cystine deposit in the cornea.

Treatment-For nearly a year, until he was two and a half years old, the patient received 100,000 units of calciferol daily, the dosage having been stepped up from 20,0001 units. Thereafter the dosage was increased to 500,000 units daily. Shohl's citric-acid-sodium-citrate solution was not given.

Progress - At the age of three years there was a trace of sugar in the urine and an excess of amino-acids, with the same chromatographic pattern as at the previous examination.

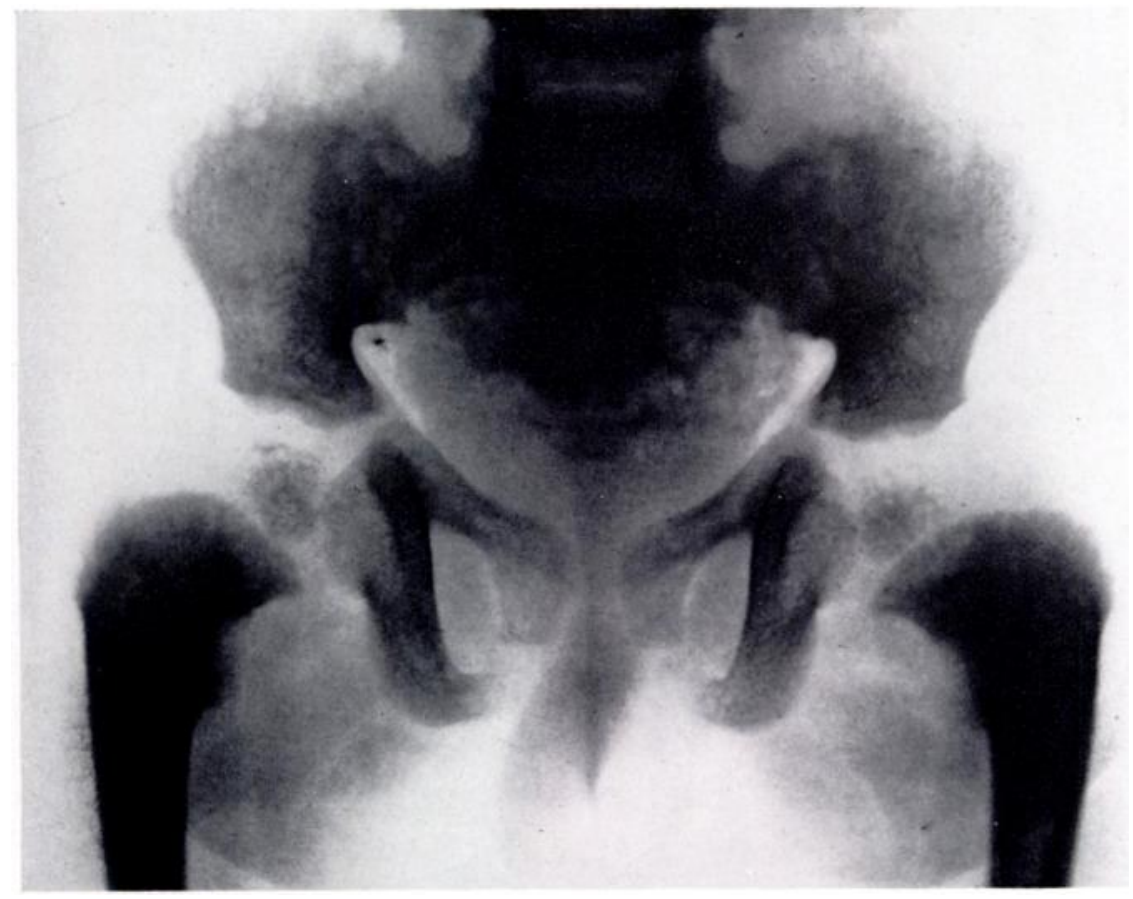

FIG. 2

Showing typical rachitic changes at the epiphyses. 
According to Dent (19:3i3) this constancy of pattern over a period of time is characteristic of Fanconi's syndrome.

Investigations, repeated at the age of three years, showed the following. Blood-serum calcium 9.2 milligrams per cent; alkaline phosphatase $\mathbf{5 7 \cdot 6}$ units; blood phosphorus 4.() milligrams per cent; $\left(\mathrm{O}_{2}\right.$ combining power 47.5 vols. Further examination at the age of four and a half revealed the following. Crine This contained no amino-acids and no sugar.

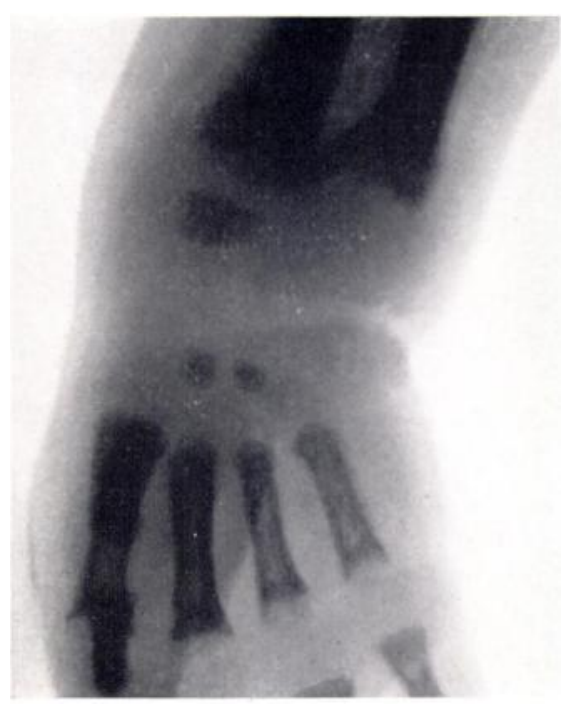

FIG. 3

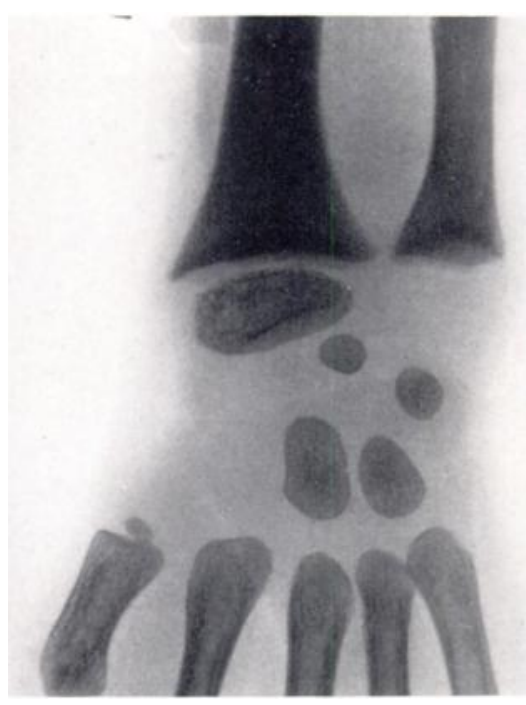

FiG. 4

Figure 3-left wrist before treatment. Figure 4-Ifter twenty months treatment with massive doses of calciferol.

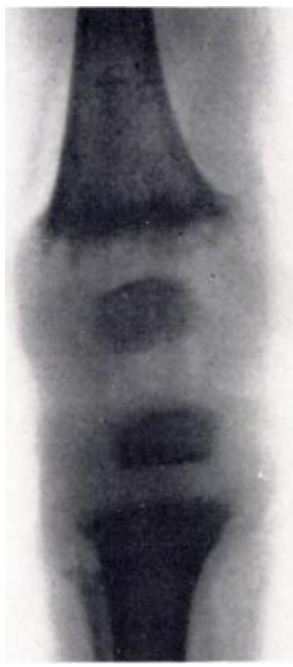

FIG. 5

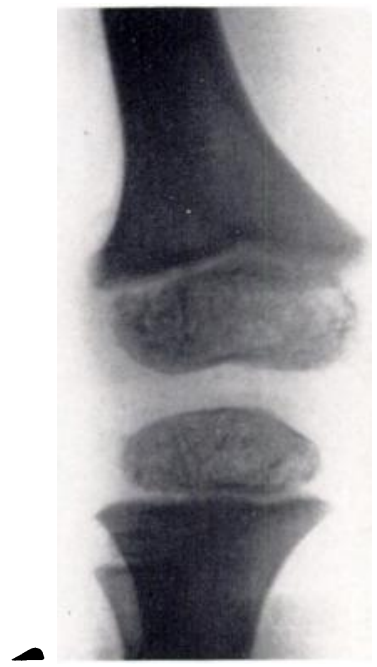

FIG. 6

Figure 5 Right knee before treatment. Figure 6 - Ifter twenty months' treatment with massive doses of calciferol.

Blood - serum calcium $9 \cdot 3$ milligrams per cent (cf. 9·: milligrams per cent) ; alkaline phosphatase $1: 5 \cdot 3$ units (cf. $7: 3$ units) ; blood phosphorus $5 \cdot()$ milligrams per cent (cf. $2 \cdot 7$ milligrams per cent); ( $\mathrm{O}_{2}$ combining power $5.3 \cdot 3$ vols. (cf. 39) vols.). At this time the radiographs showed healing (Figs. 4 and 6 ). This corresponded with the biochemical findings, namely a rise in blood phosphorus and $\mathrm{CO}_{2}$ combining power and a fall in phosphatase. At the age of five and a half the biochemical findings were quite normal.

VOL. 36 B, NO. 4, NONEMBER 1954 
Correction of deformities--At the age of four and a half vears, when there was good evidence of regression of the rachitic process, the tibial bowing was corrected by Moore's (194i) two-stage osteotomy, a technique well suited for the treatment of unusual types of deformity. Before
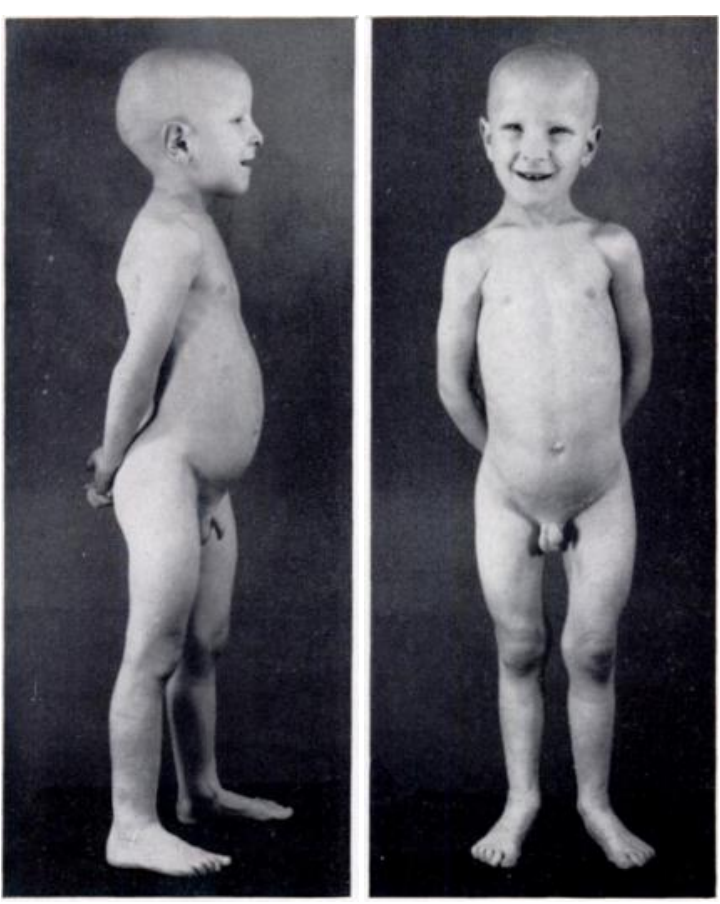

FIG. 7

Appearance after correction of tibial bowing. the operation the calciferol was stopped for two weeks. The first stage of the osteotomy was performed on the right tibia. The fibula was first exposed and divided by an osteotome, and through a separate incision the summit of the curve of the tibia was exposed subperiosteally. A wedge of bone was removed leaving the posterior cortex intact; the wedge of bone was cut into small pieces and packed into the gap. No attempt was made to correct the deformity and the limb was enclosed in plaster. Two weeks later the plaster was removed and the deformity corrected by simple manipulation under anaesthetic. At the same time the first stage was performed on the left side and after a further two weeks the second stage.

A month after the last operation the plasters were removed (Fig. 7). The calciferol therapy was restarted ten weeks after it had been stopped. At first $200,(000)$ units per day were given; this was gradually decreased to 100,000 units and at the time of writing this dose is being maintained.

The child's general condition is now excellent; he is alert and active and has a good appetite, and his weight is normal. Further examination of the urine reveals no amino-acids or glucose. There has been no recurrence of the tibial bowing.

\section{SUMMARY}

1. A case of Fanconi's syndrome, with evidence of renal tubular dysfunction, is reported.

2. Healing of the rachitic lesions followed massive doses of calciferol. There was simultaneous improvement in the biochemical state. This case is probably unique in that the amino-aciduria disappeared

3. Two-stage osteotomies were undertaken for the correction of severe bowing of the tibiae. 4. No toxic symptoms have developed so far from the calciferol theraps:

I have pleasure in recording my thanks to Mr David Trevor for permission to publish this case and to Mr K. I. Nissen and I)r C. E. Dent for their kind and helpful criticism. I would like to thank Mr R. H. Whitley for the photographs.

\section{REFERENCES}

I)ENT, (. E. (1952): Rickets and Osteomalacia from Renal Tubule Inefects. Journal of Bone and Joint Surgery, 34-B, 266.

1)ENT, C. E. (1953): Personal communication.

Fanconi, G. (1936): Der frühinfantile nephrotisch-glykosurische Zwergwuchs mit hypophosphatamischer Rachitis. Jahrbuch für Kinderheilkunde, 147, 299.

Moore, J. R. (1947): Osteotomy-Osteoclasis: a Method for Correcting Long-Bone Deformities. Journal of Bone and Joint Surgery, 29, 119. 